\title{
Transdermal Application of Drugs and Techniques Affecting Skin Barrier
}

\section{Josef Jampilek*}

Department of Chemical Drugs, Faculty of Pharmacy, University of Veterinary and Pharmaceutical Sciences, Palackeho 1:3, 61242 Brno, Czech Republic

Keywords: Drugs; Skin Barrier; Transdermal administration; Transdermal penetration; Classification of techniques

\section{Introduction}

The development in the field of pharmaceutical dosage forms results in discovery of additional highly sophisticated drug delivery systems that allow maintaining constant level of active substance in organism. Transdermal administration of drugs represents an excellent alternative to conventional pharmaceutical dosage forms. However, transdermal drug delivery often faces the problem of insufficient or no penetration of active pharmaceutical substances through the skin.

The aim of this contribution is to describe advantages and disadvantages of transdermal administration of drugs and the structure of the skin as a barrier preventing penetration of any heterogeneous substances into the body. The main part of the article is devoted to classification and brief characterization of techniques affecting the skin barrier, including up-to-date ones.

The skin consists of three basic functional layers: the upper layer (epidermis), curium (dermis), and subcutaneous tissue (hypodermis). The skin has a number of different functions; the most important is protection from excessive water loss and mechanical, chemical, microbial and physical impacts. The most important is the outermost layer of epidermis, the horny layer (stratum corneum, SC), which is in fact the skin barrier. The structure of the SC can be described as "bricks and mortar" model: corneocytes rich in keratin represent hydrophilic "bricks", and the lipid matrix represents hydrophobic "mortar". The penetration through the least permeable layer, the SC, is limiting. The epidermis acts as a barrier only to penetration of extremely lipophilic compounds. Generally, there are three main possible routes for penetration of drug molecules through the intact skin or the SC: $i)$ appendageal route (transport via sebaceous and sweat glands and the transfollicular route - through hair follicles), ii) transcellular route (through corneocytes), iii) intercellular route $[1,2]$.

At present transdermally applicable drugs include glycerol trinitrate, scopolamine, estrogens (in combination with gestagens), testosterone, fentanyl, buprenorphine, flurbiprofen, clonidine, propranolol, nicotine, rotigotine [3,4], sildenafil [5], sumatriptan [6], various (non)-steroidal anti-inflammatory drugs and antimicrobial chemotherapeutics [7]. A number of new pharmaceuticals are developed, or under clinical evaluation, for example, physostigmine [8], selegiline [9], paroxetine [10] insulin [11], and 5-fluorouracil [12]. However, the number of drugs that can be applied transdermally is limited more than it was originally expected. The use of transdermal administration is limited by some requirements on the drug. The ideal properties of a molecule that effectively penetrates through the SC include drug solubility, penetrability (drug gets into the skin), permeation (drug passing through the skin), and resorption in blood or lymphatic vessels. The applied drug dosage cannot be higher than $25 \mathrm{mg} /$ day; drug molecular weight should be $<500 \mathrm{Da}$; $\log P$ from 1 to 3 (ideal 2.5) and drug melting temperature should be less than $200^{\circ} \mathrm{C}$. Besides, the drug should not cause any skin irritation or immunity reactions. Only about $5 \%$ of drugs are suitable for administration through the skin $[13,14]$.
The advantages of transdermal administration include, above all, good pharmacokinetic properties of application systems, the ability to maintain long-lasting steady-state plasma concentration of active substances, including drugs with short biological half-lives, which reduces undesirable side effects occurring as a result of considerable fluctuations of drug plasma concentration. In contrast, the plasma levels achieved with the use of conventional dosage forms exhibit peaks and may even reach a toxic level leading to complications. Presystemic elimination of the applied dosage (hepatic first-pass effect) and effects such as $\mathrm{pH}$ change in the GIT or interactions with other simultaneously applied pharmaceutics or food are also effectively prevented here. Transdermal administration also provides possibility to apply drugs with a narrow therapeutic window and to interrupt drug delivery into the system immediately when an undesirable effect occurs (in contrast to other conventional dosage forms, which do not provide such possibility). Another important advantage is very simple and painless application. This way of administration is a non-invasive alternative to parenteral, subcutaneous, and intramuscular injections. One of the major disadvantages of transdermal application is possibility of local skin irritation or allergisation by an active substance or excipient. Other disadvantages include variable intra and interindividual absorption depending on skin conditions and, partly, on the place of application. Differences in skin structure and thickness on different body parts cause great absorption diversity. Long-term transdermal application on the same place can damage the skin by affecting its microflora and enzymes. Further disadvantage is a longer time of effect onset connected with the need to overcome the skin barrier [15].

To solve this critical issue various approaches for overcoming the skin barrier were developed. These approaches can be classified as chemical (modification of drugs, using transdermal chemical penetration enhancers) or physical (modification of drug particles size to nanosize, physical enhancement techniques). Another classification can be based on optimization of drug/vehicle or on SC modification. Optimization of drug/vehicle consists in preparation/ application of $i$ ) lipophilic prodrugs or ion-pairs, ii) eutectic systems, iii) complexes of drugs with cyclodextrins, iv) liposomes and other vesicles (transfersomes, ethosomes, niosomes, etc.), v) solid lipid nanoparticles and other nanoparticles and/or nanodelivery systems, vi) saturated and supersaturated solutions. Modification, i.e., hydration/lipid fluidization/disrupting of SC, means $i$ ) application of transdermal chemical penetration enhancers, $i$ ) overall optimization

*Corresponding author: Josef Jampilek, Department of Chemical Drugs Faculty of Pharmacy, University of Veterinary and Pharmaceutical Sciences, Palackeho 1:3, 61242 Brno, Czech Republic, Tel: +420541652926; E-mail: josef.jampilek@gmail.com

Received May 10, 2013; Accepted September 23, 2013; Published September 30,2013

Citation: Jampilek J (2013) Transdermal Application of Drugs and Techniques Affecting Skin Barrier. J Bioequiv Availab 5: 233-235. doi:10.4172/jbb.1000164

Copyright: (c) 2013 Jampilek J. This is an open-access article distributed under the terms of the Creative Commons Attribution License, which permits unrestricted use, distribution, and reproduction in any medium, provided the original author and source are credited. 
of formulation using non-hydrophobic excipients or iii) application of physical enhancement techniques (electrically assisted methods), such as iontophoresis, electroporation, acoustic methods - ultrasound (phonophoresis, sonophoresis), microneedles, magnetophoresis or photomechanical wave $[2,7,10,14-23]$.

The disadvantage of these methods is their relatively complicated use requiring special apparatuses for application and their cost. A number of authors mention combination of these physical methods with the use of chemical penetration enhancers as a very effective method of transdermal drug application [16,24]. Ganga et al. [25] described a synergistic effect of Azone and iontophoresis on penetration of metoprolol through human epidermis in vitro. The synergistic effect of ultrasound and chemical substances was demonstrated by the use of polyethylene glycol, isopropyl myristate, linoleic acid or sodium lauryl sulphate [26,27]. Several authors mention a combined effect of electroporation and macromolecular substances which should increase and stabilize formed pores [28,29]. Iontophoresis, electroporation, acoustical methods and microneedles that show considerable promise are briefly mentioned below.

Iontophoresis is a physical method using a low-level direct current to deliver substances through the intact skin. Typically a few milliamperes of current (15-30 mA) are applied to a few square centimetres of the skin, which generally causes no pain or irritation [30]. A drug reservoir is placed on the skin under the active electrode with the same charge as the penetrating drug, and an indifferent counter electrode is placed elsewhere on the body. The active electrode repels the drug molecules and forces them into the skin. The quantity of molecules crossing the barrier correlates directly with the applied current. Iontophoresis can facilitate substance transport through the skin by a number of possible mechanisms, including an electrophoretic driving force and an electroosmotic driving force causing an increase of skin permeability [31]. Extensive studies mention the possibility of the use of iontophoresis for a wide range of pharmaceuticals including antiinflammatory compounds, hydrophilic molecules, hormones and macromolecular compounds [32]. The U.S. Food and Drug Administration (FDA) approved sumatriptan iontophoretic transdermal system for acute migraine treatment of adults [6].

Electroporation temporarily increases skin permeability by short-term application of high-voltage pulses to the skin. In contrast to iontophoresis, where low voltage is used, electroporation requires higher voltage within short-time interval from $10 \mu$ s to $100 \mathrm{~ms}$. Electroporation creates micropores in the lipid bilayer, enabling penetration of hydrophilic substances and macromolecules [33]. Among the compounds studied in connection with the use of electroporation for transdermal application, e.g. heparin [34], insulin [35], and vaccines [36].

For facilitation of drug transport through the skin, ultrasonic and short-duration shock waves can also be used. The method using different frequencies of ultrasound from $20 \mathrm{kHz}$ to $16 \mathrm{MHz}$ for the improvement of skin permeability is designated as sonophoresis [37]. Ultrasonic waves affect the skin by a number of mechanisms. One of the effects is formation, oscillation, and sequent collapse of gaseous bubbles (gasfilled cavities) in the liquid. This process indicated as cavitation results in formation of holes in corneocytes, enlargement of the intercellular space and perturbation of SC lipid bilayers. Also thermal effect as a result of ultrasound absorption by the skin is observed. There is usually temperature increase within several degrees; it enhances lipid fluidity and molecule diffusion through the skin barrier [38,39]. Numerous authors mention application of sonophoresis for enhancement of skin permeability for different pharmaceuticals, including insulin, erythropoietin, $\gamma$-interferon, and heparin [40,41].

Needles of microscopic size (microneedles) can penetrate into the skin surface and create holes large enough for drug molecules to enter but simultaneously small enough to avoid pain and significant damage to the skin. The length of microneedles connected to a reservoir is within $10-200 \mu \mathrm{m}$; they can be solid or hollow, with a channel through which drug solutions flow into the skin. Microneedles penetrate through the SC and epidermis without reaching the upper layer of the dermis where nerve endings are situated. Experiments conducted in vitro demonstrated that small drug molecules, big macromolecules and nanoparticles can be administered using this method [42-45].

The problematics of transdermal application of drugs, transdermal therapeutic systems and methods of overcoming the skin barrier is extensive, and, of course, it is not possible to cover all aspects in this short article. Nevertheless, after a certain stagnation, transdermal drug application is currently extensively investigated as a perspective noninvasive drug administration method. The subject of this short review was brief but comprehensive introduction of this interesting problematics bridging medicinal chemistry, pharmaceutical technology and biophysics with emphasis on the use of physical enhancement techniques.

\section{Acknowledgments}

This study was supported by the Czech Science Foundation; project number GACR P304/11/2246.

\section{References}

1. Freinkel RK, Woodley DT (2001) The biology of the skin. Parthenon Publishing.

2. Jampilek J, Brychtova K (2012) Azone analogues: classification, design, and transdermal penetration principles. Med Res Rev 32: 907-947.

3. Swart PJ, Toulouse FAM, De Zeeuw RA (1992) The influence of Azone on the transdermal penetration of the dopamine D2 agonist $\mathrm{N}-0923$ in freely moving rats. Int J Pharm 88: 165-170.

4. Muller W, Peck JV (2008) Transdermal therapeutic system for treating parkinsonism. US Patent 7413747.

5. Elnaggar YSR, El-Massik MA, Abdallah OY (2011) Sildenafil citrate nanoemulsion vs. self-nanoemulsifying delivery systems: rational development and transdermal permeation. Int J Nanotechnol 8: 749-763.

6. Barclay L (2013) FDA approves transdermal patch for migraine. Medscape Today News.

7. Jampilek J, Opatrilova R, Coufalova L, Cernikova A, Dohnal J (2013) Utilization of alaptide as transdermal penetration modifier in pharmaceutical compositions for human and veterinary applications containing anti-inflammatory drugs and/ or antimicrobial chemotherapeutics. WO/2013/020527 A1.

8. Moller HJ, Hampel H, Hegerl U, Schmitt W, Walter K (1999) Double-blind, randomized, placebo-controlled clinical trial on the efficacy and tolerability of a physostigmine patch in patients with senile dementia of the Alzheimer type. Pharmacopsychiatry 32: 99-106.

9. Lee KC, Chen JJ (2007) Transdermal selegiline for the treatment of major depressive disorder. Neuropsychiatr Dis Treat 3: 527-537.

10. El-Nabarawi MA, Bendas ER, El Rehem RT, Abary MY (2013) Transdermal drug delivery of paroxetine through lipid-vesicular formulation to augment its bioavailability. Int J Pharm 443: 307-317.

11. Wong TW (2009) Chitosan and its use in design of insulin delivery system. Recent Pat Drug Deliv Formul 3: 8-25.

12. Chandrashekar NS, Prasanth VV (2008) Clinical evaluation of 5-fluorouracil from transdermal patches on EAC and DLA cell-induced tumors in mice. Asian Pac J Cancer Prev 9: 437-440.

13. Finnin BC, Morgan TM (1999) Transdermal penetration enhancers: applications, limitations, and potential. J Pharm Sci 88: 955-958. 
Citation: Jampilek J (2013) Transdermal Application of Drugs and Techniques Affecting Skin Barrier. J Bioequiv Availab 5: 233-235. doi:10.4172/ jbb.1000164

14. Benson HA (2005) Transdermal drug delivery: penetration enhancement techniques. Curr Drug Deliv 2: 23-33.

15. Vasilev AE, Krasnyuk II, Ravikumar S, Tokhmakhchi VN (2001) Transdermal therapeutic systems for controlled drug release. Pharm Chem J 35: 613-626.

16. Barry BW (2001) Novel mechanisms and devices to enable successful transdermal drug delivery. Eur J Pharm Sci 14: 101-114.

17. Karande P, Mitragotri S (2009) Enhancement of transdermal drug delivery via synergistic action of chemicals. Biochim Biophys Acta 1788: 2362-2373.

18. Jampilek J, Opatrilova R, Rezacova A, Oktabec Z, Dohnal J (2012) Alaptide: Methods of effecting its solubility, membrane permeation and pharmaceutical compositions for human and veterinary applications. PTC/CZ2012/000074.

19. Singla S, Harikumar SL, Aggarwal G (2012) Proniosomes for penetration enhancement in transdermal system. Int J Drug Dev Res 4: 1-13.

20. Leite-Silva VR, de Almeida MM, Fradin A, Grice JE, Roberts MS (2012) Delivery of drugs applied topically to the skin: Intrinsic skin properties affecting drug delivery. Exp Rev Dermatol 7: 383-397.

21. Fang JY, Leu YL (2006) Prodrug strategy for enhancing drug delivery via skin. Curr Drug Discov Technol 3: 211-224.

22. Baroli B (2010) Penetration of nanoparticles and nanomaterials in the skin fiction or reality? J Pharm Sci 99: 21-50.

23. Prow TW, Grice JE, Lin LL, Faye R, Butler M, et al. (2011) Nanoparticles and microparticles for skin drug delivery. Adv Drug Deliv Rev 63: 470-491.

24. Mitragotri S (2000) Synergistic effect of enhancers for transdermal drug delivery. Pharm Res 17: 1354-1359

25. Ganga S, Ramarao J, Singh J (1996) Effect of Azone on the iontophoretic transdermal delivery of metoprolol tartarate through human epidermis in vitro. J Control Release 42: 57-64.

26. Johnson ME, Mitragotri S, Patel A, Blankschtein D, Langer R (1996) Synergistic effects of chemical enhancers and therapeutic ultrasound on transdermal drug delivery. J Pharm Sci 85: 670-679.

27. Mitragotri S, Ray D, Farrell J, Tang H, Yu B, et al. (2000) Synergistic effect of low-frequency ultrasound and sodium lauryl sulfate on transdermal transport. J Pharm Sci 89: 892-900.

28. Vanbever R, Prausnitz MR, Preat V (1997) Macromolecules as novel transdermal transport enhancers for skin electroporation. Pharm Res 14: 638644.

29. Zewert TE, Pliquett UF, Vanbever R, Langer R, Weaver JC (1999) Creation of transdermal pathways for macromolecule transport by skin electroporation and a low toxicity, pathway-enlarging molecule. Bioelectrochem Bioenerg 49 : $11-20$
30. Ledger PW (1992) Skin biological issues in electrically enhanced transderma delivery. Adv Drug Deliv Rev 9: 289-307.

31. Pikal MJ (2001) The role of electroosmotic flow in transdermal iontophoresis Adv Drug Deliv Rev 46: 281-305.

32. Riviere JE, Heit MC (1997) Electrically-assisted transdermal drug delivery. Pharm Res 14: 687-697.

33. Prausnitz MR (1999) A practical assessment of transdermal drug delivery by skin electroporation. Adv Drug Deliv Rev 35: 61-76.

34. Prausnitz MR, Edelman ER, Gimm JA, Langer R, Weaver JC (1995) Transdermal delivery of heparin by skin electroporation. Biotechnology (N Y) 13: $1205-1209$.

35. Sen A, Daly ME, Hui SW (2002) Transdermal insulin delivery using lipid enhanced electroporation. Biochim Biophys Acta 1564: 5-8.

36. Misra A, Ganga S, Upadhyay P (1999) Needle-free, nonadjuvanted skin immunization by electroporation-enhanced transdermal delivery of diphtheria toxoid and a candidate peptide vaccine against hepatitis $B$ virus. Vaccine 18 517-523.

37. Mitragotri S, Blankschtein D, Langer R (1996) Transdermal drug delivery using low-frequency sonophoresis. Pharm Res 13: 411-420.

38. Ueda H, Mutoh M, Seki T, Kobayashi D, Morimoto Y (2009) Acoustic cavitation as an enhancing mechanism of low-frequency sonophoresis for transdermal drug delivery. Biol Pharm Bull 32: 916-920.

39. Ogura M, Paliwal S, Mitragotri S (2008) Low-frequency sonophoresis: current status and future prospects. Adv Drug Deliv Rev 60: 1218-1223.

40. Smith NB, Lee S, Shung KK (2003) Ultrasound-mediated transdermal in vivo transport of insulin with low-profile cymbal arrays. Ultrasound Med Biol 29 1205-1210.

41. Mitragotri S, Blankschtein D, Langer R (1995) Ultrasound-mediated transderma protein delivery. Science 269: 850-853.

42. Henry S, McAllister DV, Allen MG, Prausnitz MR (1998) Microfabricated microneedles: a novel approach to transdermal drug delivery. J Pharm Sci 87 922-925.

43. McAllister DV, Wang PM, Davis SP, Park JH, Canatella PJ, et al. (2003) Microfabricated needles for transdermal delivery of macromolecules and nanoparticles: fabrication methods and transport studies. Proc Natl Acad Sci U S A 100: 13755-13760.

44. Donnelly RF, Majithiya R, Singh TR, Morrow DI, Garland MJ, et al. (2011) Design, optimization and characterisation of polymeric microneedle arrays prepared by a novel laser-based micromoulding technique. Pharm Res 28: 4157.

45. Bariya SH, Gohel MC, Mehta TA, Sharma OP (2012) Microneedles: an emerging transdermal drug delivery system. J Pharm Pharmacol 64: 11-29. 